\title{
Femtosecond X-ray Absorption Spectroscopy at a Hard X-ray Free Electron Laser: Application to Spin Crossover Dynamics
}

Henrik T. Lemke, ${ }^{\dagger}$ Christian Bressler, ${ }^{\ddagger}$ Lin X. Chen ${ }^{\S}$ David M. Fritz, ${ }^{\dagger}$ Kelly J. Gaffney, ${ }^{\perp}$ Andreas Galler, ${ }^{\ddagger}$ Wojciech Gawelda, ${ }^{\ddagger}$ Kristoffer Haldrup, ${ }^{\#}$ Robert W. Hartsock, ${ }^{\perp}$ Hyotcherl Ihee, ${ }_{s}^{\text {II } \$}$ Jeongho Kim, ${ }^{@}$ Kyung Hwan Kim, ${ }^{\text {I, } \$ \text { Jae Hyuk Lee, }}{ }^{\$}$ Martin M. Nielsen, ${ }^{\#}$ Andrew B. Stickrath, ${ }^{\S}$ Wenkai Zhang, ${ }^{\perp}$ Diling Zhu, ${ }^{\dagger}$ and Marco Cammarata ${ }^{*} \dagger, \Delta$

${ }^{\dagger}$ LCLS, SLAC National Laboratory, Menlo Park, California 94025, United States

${ }^{\ddagger}$ European XFEL, Albert-Einstein-Ring 19, 22761 Hamburg, Germany

${ }^{\S}$ Chemical Sciences and Engineering Division, Argonne National Laboratory, Lemont, Illinois 60439, United States

"Department of Chemistry, Northwestern University, Evanston, Illinois 60208, United States

${ }^{\perp}$ Pulse Institute, SLAC National Laboratory, Menlo Park, California 94025, United States

${ }^{\#}$ Centre for Molecular Movies, Department of Physics, NEXMAP Section, Technical University of Denmark, 2800 Kgs. Lyngby, Denmark

${ }^{\text {II }}$ Center for Nanomaterials and Chemical Reactions, Institute for Basic Science, Daejeon 305-701, Republic of Korea

${ }^{\circledR}$ Department of Chemistry, Inha University, Incheon 402-751, Republic of Korea

${ }^{\$}$ Center for Time-Resolved Diffraction, Department of Chemistry, KAIST, Daejeon 305-701, Republic of Korea

${ }^{\Delta}$ Institut de Physique de Rennes, UMR UR1-CNRS 6251, Université de Rennes 1, F35042, Rennes, France

ABSTRACT: X-ray free electron lasers (XFELs) deliver short $(<100 \mathrm{fs})$ and intense $\left(\sim 10^{12}\right.$ photons) pulses of hard X-rays, making them excellent sources for time-resolved studies. Here we show that, despite the inherent instabilities of current (SASE based) XFELs, they can be used for measuring high-quality X-ray absorption data and we report femtosecond time-resolved Xray absorption near-edge spectroscopy (XANES) measurements of a spin-crossover system, iron(II) tris $\left(2,2^{\prime}\right.$-bipyridine $)$ in water. The data indicate that the low-spin to high-spin transition can be modeled by single-exponential kinetics convoluted with the overall time resolution. The resulting time constant is $\sim 160 \mathrm{fs}$.

\section{INTRODUCTION}

Chemical reactions involve the dynamic evolution of strongly coupled electrons and nuclei on ultrafast time scales from few to a few hundred femtoseconds. Disentangling this complex dynamics has motivated a wide range of experimental and theoretical studies to obtain a more complete picture of the elementary steps underlying these processes. ${ }^{1}$ Despite the progresses in ultrafast optical spectroscopies that can resolve dynamics of the valence shell electrons with femtosecond time resolution and in theoretical calculations of many-body dynamics, our understanding of the early chemical transformations still remains incomplete due to the lack of direct structural measurements visualizing both electronic structure and nuclear movements in real time. Information on nuclear dynamics has been obtained with time-resolved scattering with a time resolution of $100 \mathrm{ps}$ on a variety of system from solutions $^{2,3}$ to single crystals. ${ }^{4-6}$ Recently, femtoseconds studies have been published. ${ }^{7-9}$

When the surrounding of specific atoms is of interest, recent advances have demonstrated the utility of time-resolved X-ray absorption spectroscopy (XAS) as a reliable monitor of nuclear and electronic structure during chemical reactions on both the picosecond ${ }^{10,11}$ and, more recently, the femtosecond time scale. ${ }^{12-14}$ Yet, most of the synchrotron-based experiments are limited to insufficient time resolution $(\sim 100$ ps in normal operating conditions) or very low flux ( $\sim 10$ photons/pulse at kilohertz rate, when operated in femtosecond laser slicing mode $^{15}$ ). The development of new X-ray facilities such as X-ray free electron lasers (XFELs) provide unprecedented capabilities in terms of both X-ray pulse durations (down to tens of femtoseconds) and single pulse intensities of more than $10^{10}$ photons when a $1 \mathrm{eV}$ bandwidth monochromator is used. In the present study we exploit XFEL radiation from the Linac Coherent Light Source (LCLS), the first hard XFEL, ${ }^{16}$ and present an experimental study of the photoexcited spin transition dynamics in aqueous ferreous bipyridine ([Fe$\left.\left.(\text { bpy })_{3}\right]^{2+}\right)$, which proves the feasibility of femtosecond XAS at the LCLS.

Transition metal complexes exhibiting photoinduced spin crossover (SCO) transition provide a good example of the electronic dynamics coupled with nuclear motions. In a

Received: December 20, 2012

Published: January 2, 2013 
prototypical SCO compound, $\left[\mathrm{Fe}(\mathrm{bpy})_{3}\right]^{2+}$ (Figure 1), three bipyridine (bpy) ligands generate a ligand field, which splits the
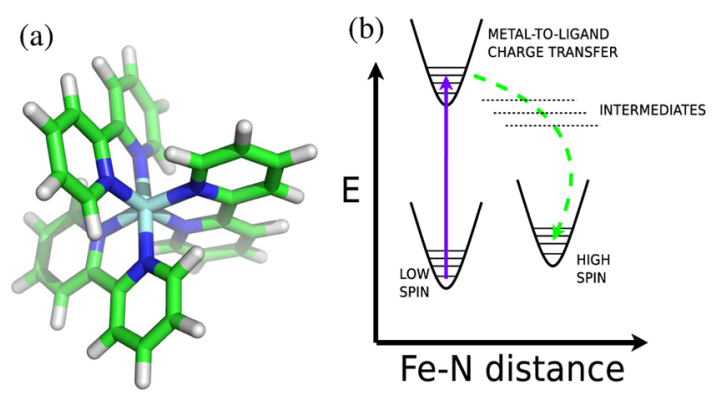

Figure 1. (a) Geometric model of the $\left[\mathrm{Fe}(\mathrm{bpy})_{3}\right]^{2+}$ molecule. The central atom (cyan) is $\mathrm{Fe}$, surrounded by the nearest neighboring $\mathrm{N}$ atoms (dark blue), $\mathrm{C}$ atoms (green) and $\mathrm{H}$ atoms (light gray), which constitute the bipyridine ligands. (b) Simplified photocycle diagram of $\left[\mathrm{Fe}(\mathrm{bpy})_{3}\right]^{2+}$. Upon the optical laser absorption (blue vertical arrow) the molecule is excited to the so-called metal-to-ligand charge transfer (MLCT) manifold, where one electron from the $\mathrm{Fe}$ is transferred to the bpy ligands. The relaxation processes from MLCT band to the lowest lying high-spin ${ }^{5} \mathrm{~T}_{2}$ state (dashed green line) may involve some intermediate ligand-field states.

$\mathrm{Fe}$ (II) $3 \mathrm{~d}$ molecular orbitals into $3 \mathrm{~d}\left(\mathrm{t}_{2 \mathrm{~g}}\right)^{6}$ at lower energy and $3 \mathrm{~d}\left(\mathrm{e}_{\mathrm{g}}\right)^{0}$ at higher energy, which are respectively fully occupied and empty in the low-spin (LS) ground state. ${ }^{17}$ The stability of the LS state depends critically on the distance of the bpy ligands, where expansion of the $\mathrm{Fe}-\mathrm{N}$ coordination bonds by $\sim 10 \%$ makes the high-spin (HS) quintet state the lowest energy configuration. ${ }^{18}$ Due to the rather large energy difference between LS and HS states $(0.6 \mathrm{eV}$ for the compound presented here), only photoexcitation can lead to an efficient conversion to the HS states. ${ }^{19}$ Ultrafast optical transient absorption spectroscopy has been used to investigate the dynamics of iron-based SCO complexes by photoexciting their metal-to-ligand charge transfer (MLCT) absorption bands centered around $520 \mathrm{~nm}$, which results in shifting the electron density from $\mathrm{Fe}$ (II) center to the ligands. It has been previously reported that the photoexcitation of the MLCT absorption bands triggers a cascade of spin crossover transitions, eventually populating the lowest energy HS state with near-unity quantum yield. ${ }^{20}$ Transient absorption spectroscopy and femtosecond fluorescence studies have shown that intersystem crossing (ISC) within the MLCT manifold is quite fast $\left(\sim 30 \mathrm{fs}^{21}\right)$ and population of the HS state occurs in $<1 \mathrm{ps}^{22,23}$ Although the ultrafast ISC appears to be a common property of the ferrous iron coordination complexes, the mechanism for SCO still remains unclear, in particular regarding the role of possible intermediate ligand-field states between the MLCT and the HS state (so-called ${ }^{1} \mathrm{~T}_{1},{ }^{3} \mathrm{~T}_{1},{ }^{3} \mathrm{~T}_{2}$, and ${ }^{1} \mathrm{~T}_{2}$ ). ${ }^{24}$ This uncertainty is difficult to address using the ultrafast transient optical absorption spectroscopy, because the transitions to the involved intermediate spin states are all spin-forbidden and buried throughout the UV-vis range by various allowed charge transfer transitions involving metal and ligand (i.e., MLCT) or ligands only. ${ }^{25}$ In contrast, time-resolved XAS makes use of the X-ray absorption properties/cross section of the atomic core electrons as an element-selective probe of the local geometrical and electronic structure, in particular the oxidation state of the absorber. Therefore, in principle, time-dependent changes of the nuclear structure and the oxidation state can be extracted from the transient XAS spectra in a robust manner and can thus complement the ultrafast optical measurements.

\section{EXPERIMENTAL METHODS}

We performed the femtosecond X-ray absorption near edge structure (XANES) measurements on a $50 \mathrm{mM}$ aqueous solution of $\left[\mathrm{Fe}(\mathrm{bpy})_{3}\right] \mathrm{Cl}_{2}$ at the XPP station of the LCLS (Figure 2). The experiment used a $0.1 \mathrm{~mm}$ thick liquid jet

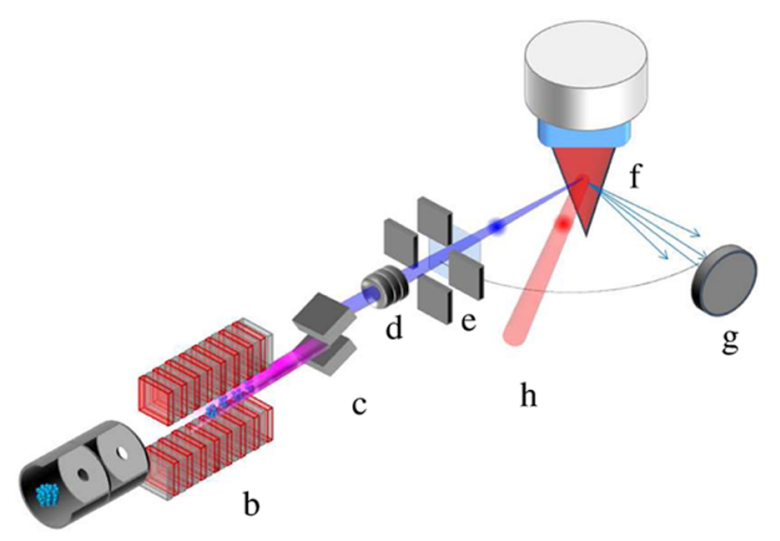

a

Figure 2. Schematic representation of the experimental layout: (a) accelerator section, (b) $100 \mathrm{~m}$ long undulator generating short $(\sim 30$ fs) and intense ( $\sim 10^{12}$ photons per pulse) X-ray pulses, (c) double crystal monochromator, (d) X-ray focusing Be lenses, (e) noninvasive intensity monitor (the one before the monochromator is not shown), (f) free-flowing liquid jet containing an aqueous solution of $\left[\mathrm{Fe}(\mathrm{bpy})_{3}\right]^{2+}$, (g) X-ray diode measuring the total fluorescence yield signal, and (h) pump laser beam, with significantly exaggerated angle between the laser and X-ray beam propagation directions. During the experiment, the angle between the laser pump and the X-ray probe was $\sim 1^{\circ}$.

oriented at an angle of $45^{\circ}$ with respect to the direction of the incident X-ray beam. The sample solution was collinearly excited with $400 \mathrm{~nm}$ laser light generated by frequency doubling of the output of a Ti:sapphire regenerative amplifier laser system (Coherent, Legend). The optical pump pulses had a pulse duration of $50 \mathrm{fs}$ (fwhm), pulse energy of $220 \mu \mathrm{J}$, and a focal area of $220 \times 150 \mu \mathrm{m}^{2}$ at the sample. The X-ray beam was monochromatized by using a $\mathrm{Si}(111)$ double-crystal monochromator and focused downstream the sample using $\mathrm{Be}$ lenses; the beamsize at the sample position was $100 \mu \mathrm{m}$ (fwhm). The estimated number of photons per pulse after the monochromator is on the order of $\sim 3 \times 10^{10}$ for the intense shots (corresponding to $6 \times 10^{9}$ photons per pulse on the sample due to $1 \mathrm{~m}$ air path before the sample).

We detected the total X-ray fluorescence yield to obtain the XANES spectra in the vicinity of the Fe K-edge $(7112 \mathrm{eV})$. The $\mathrm{X}$-ray fluorescence was collected using a Si diode (Canberra FD450-18-300RM) positioned at a $90^{\circ}$ angle with respect to the X-ray beam propagation direction. The incoming intensity of polychromatic ("white") and monochromatic beams were measured using two noninvasive diagnostics (one before and one after the X-ray monochromator) developed for pulsed Xray radiation. ${ }^{26}$ For strong shots the readout voltage of about 1 $\mathrm{V}$ has been observed corresponding to $\sim 10^{5}$ photons. ${ }^{26}$ The readout noise was of the order of few millivolts. Considering that the fluorescence diode was covering about $1 \%$ of the $4 \pi$ solid angle, the total number of fluorescence photons is $\sim 10^{7}$ 
per pulse. The timing jitter between the pump and probe pulses was estimated to be between 100 and $200 \mathrm{fs}$ fwhm (and around 300-500 fs during spectral scans, Figure 5).

Unlike storage ring synchrotron sources, XFELs use each electron bunch only once to generate X-rays before dumping the bunch and the self-amplified spontaneous emission (SASE) process underlying the X-ray generation of currently available XFEL sources is an inherently stochastic process. ${ }^{27}$ Therefore, XFELs are intrinsically less stable than storage ring based X-ray sources. This "intrinsic instability" results in large variation of both the X-ray intensity and the spectrum for consecutive X-ray pulses. A histogram for the pulse intensity of 1000 consecutive shots, which were sampled noninvasively before the monochromator, is shown in Figure 3a (red filled bars). The

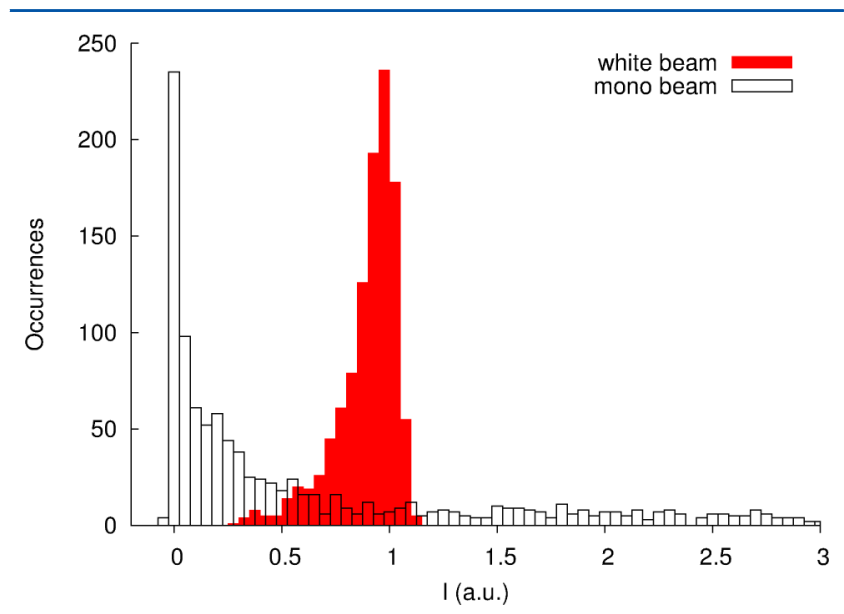

Figure 3. Histograms of 1000 consecutive X-ray pulse intensities before ("white beam", filled red bars) and after ("mono beam", empty bars) passing the X-ray monochromator. The distributions show how the intensity fluctuations are greatly amplified after the monochromator due to the energy jitter in the LCLS accelerator and the spiky nature of the SASE-based XFEL radiation.

fluctuations can be quantified by the ratio of the standard deviation over the average intensity value $\left(\sigma_{I} / \bar{I}\right)$ that resulted in $\sim 15 \%$. Due to the rather broad bandwidth $(\sim 0.3 \%$, i.e., $\sim 20$ $\mathrm{eV}$ at $7 \mathrm{keV}$ ) and shot-to-shot jitter of the central wavelength ( $\sim 20 \mathrm{eV}$ during the experiment reported here), XANES experiments require the use of an X-ray monochromator to precisely define the incoming X-ray wavelength.

Due to electron energy jitter in the LCLS accelerator and the spiky SASE spectrum, the intensity fluctuations of the monochromatic beam are enhanced with respect to the "white" beam as shown in Figure 3 (empty bars). The intensity fluctuations $\left(\sigma_{I} / \bar{I}\right)$ for the experiment discussed here are of the order of $125 \%$. $^{28}$

Despite such large intensity fluctuations of the monochromatic beam, proper normalization can be obtained after correction for diode nonlinearity. This has been achieved by taking "calibration runs" before acquiring the time-resolved data. The data, $I_{\text {fluo }}$ vs $I_{\text {mono }}$ have then been fitted to a secondorder polynomial. As can be seen in the Figure 4a, the data at higher intensity indicate a non linearity in the monitor (the second order coefficient is positive). Then calling $p_{2}$ and $p_{1}$ the second and first-order term of the polynomial, then the $I_{\text {mono }}$ can be corrected using the relation $I_{\text {mono }}^{\prime}=I_{\text {mono }}+\left(p_{2} / p_{1}\right) I_{\text {mono }}$. Figure $4 \mathrm{~b}$ shows how the linearity is greatly improved by applying such correction. The normalized fluorescence signal is
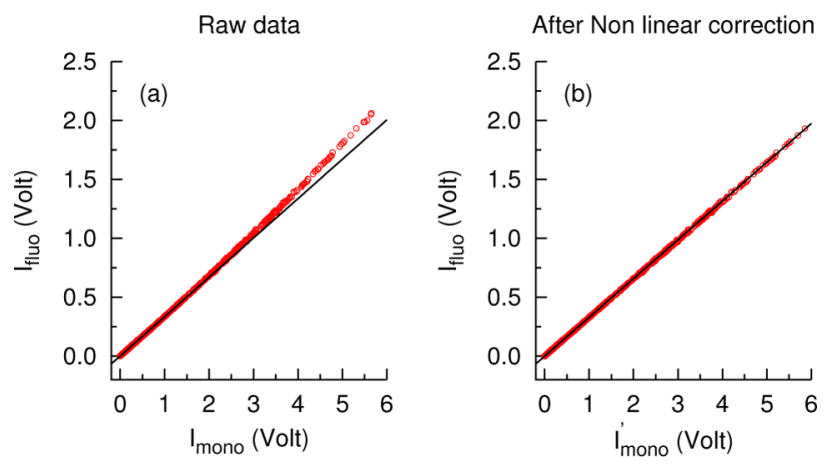

Figure 4. Correlation of the fluorescent diode readings versus incoming monochromatic intensity for the same shots shown in Figure 3. (a) Raw data together with a linear fit done for $I_{\text {mono }}<1 \mathrm{~V}$. (b) As in (a) but data have been corrected for detector nonlinearity as described in the text.

then calculated for a given "scan point" (i.e., fixed energy and time delay) by $I_{\text {norm }}=\left(\sum_{i=1}^{N} I_{\text {fluo }}^{i} / \sum_{i=1}^{N} I_{\text {mono }}^{\prime i}\right)$ where the index $i$ is used to distinguish the $N$ shots collected for a given scan point (usually around 240). Only shots for which $I_{\text {mono }}>0.1 \mathrm{~V}$ (corresponding to $\sim 20 \%$ percentile) have been retained for the calculation of the averaged normalized intensity. Error bars have been calculated using the standard deviation of the mean of the ratios $I_{\text {fluo }}^{i} / I_{\text {mono }}^{\prime i}$ for the retained shots.

We acquired spectra in the energy range $7105-7150 \mathrm{eV}$ with $0.5 \mathrm{eV}$ energy steps for time delays of -1 to +4 ps between the excitation laser pulse and the X-ray laser pulse. The difference XANES spectra were obtained from the experimental XANES spectra by subtracting signal measured at a negative time delay (X-ray pulse arriving before the optical laser pulse and thus probing the LS ground state only) from a signal at various positive time delays (after the optical laser excitation), Figure 5 a. Scans were either performed at a fixed time delay varying the X-ray photon energy (Figure 5a, "spectral scan") or at a fixed X-ray photon energy varying the time delay between the laser pump and the X-ray probe pulses (Figure 5b, "time scan"). Each point in a spectral/time scan was averaged over 120 or 240 shots (i.e., 2 or $4 \mathrm{~s}$ because LCLS was running at $60 \mathrm{~Hz}^{29}$ ). Moreover, it should be noted that the rotary motion of the monochromator adds a systematic energy dependent change of $\mathrm{X}$-ray path length by the amount of $2 h \sin (\theta)$ where $h$ is the channel size in the monochromator and $\theta$ the bragg angle. In our experimental setup $(\mathrm{h}=3.9 \mathrm{~nm})$, the change of $X$-ray path length induced by the monochromator corresponds to $48 \mathrm{fs}$ in time over the entire energy range used in our experiment.

\section{RESULTS}

As shown in Figure 5a, the shape of the difference spectra, to first approximation, does not vary with the time delay. The spectrum at the longest measured delay in this data set $(1.25$ ps) strongly resembles the one previously measured for the ${ }^{5} \mathrm{~T}_{2}$ high-spin state at time delays $\geq 50 \mathrm{ps}^{11}$ (superimposed in Figure $5 \mathrm{a}$ as solid curve). Hence we assign this transient signal to the ${ }^{5} \mathrm{~T}_{2}$ HS excited state. The difference spectrum exhibits a peak at $7125.5 \mathrm{eV}$, which has been reported to be strongly correlated with the change in $\mathrm{Fe}-\mathrm{N}$ bond distance. ${ }^{12} \mathrm{We}$ utilize the time dependent rise in transient signal at $7125.5 \mathrm{eV}$, Figure $5 \mathrm{~b}$, to monitor the rate for the formation of bond-elongated excited states like, e.g., the ${ }^{5} \mathrm{~T}_{2}$. The time dependence of the signal is clearly asymmetric around time zero, exhibiting a fast rise followed by a slower increase. This asymmetric temporal 

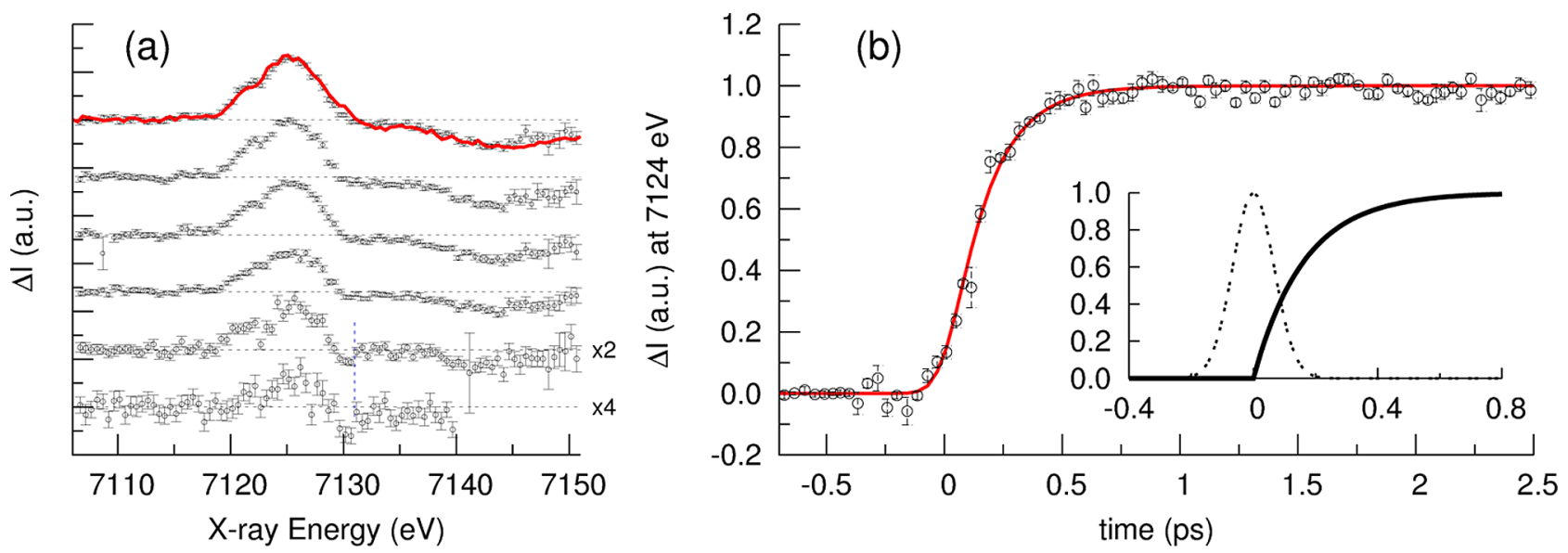

Figure 5. (a) Difference XANES spectra (empty circles) of $\left[\mathrm{Fe}(\mathrm{bpy})_{3}\right]^{2+}$ taken at time delays of $-170 \mathrm{fs}, 0 \mathrm{fs}, 190 \mathrm{fs}, 370 \mathrm{fs}, 550 \mathrm{fs}$, and $1.25 \mathrm{ps}(\mathrm{from}$ bottom to top, the scale of the bottom spectra have been vertically expanded by factors of 4 and 2, respectively). The previously measured transient XANES difference signal at $50 \mathrm{ps}^{11}$ time delay was scaled and superimposed on the spectrum at the longest time delay (red solid line); the vertical line indicates the isosbestic point for the LS-HS transition. The disappearance of the negative peak and a shift of the zero-crossing toward the LS-HS isosbestic point can be seen with increasing delay. (b) Time-dependent amplitude change of the feature at $7125.5 \mathrm{eV}$ (open circles), where the largest transient feature is observed in the difference XANES spectra. The data were fitted by convolving a temporal instrument response function (Gaussian) with an exponential rise (with a time constant $\tau$ ). The resulting fit is shown as a red solid line. The fitting parameters are $\tau=163 \pm 6 \mathrm{fs}$ and Gaussian fwhm $=155 \pm 16$ fs. The inset of panel (b) shows the instrument response function (dashed line) function and the exponential rise time (solid line) resulting from the fitting procedure.

behavior indicates that our measurement has sufficient time resolution to measure the rate of the metastable ${ }^{5} \mathrm{~T}_{2}$ state formation. A phenomenological fit of the transient signal to a convolution of a single exponential rise with a Gaussian instrument response function gives $155 \pm 16 \mathrm{fs}$ fwhm overall instrument response function and an exponential rise time of $163 \pm 6$ fs. The error values reported here refer to one standard deviation. The time resolution is close to the optimal one for the experimental geometry, where the $0.1 \mathrm{~mm}$ jet thickness at $45^{\circ}$ leads to a $\sim 130 \mathrm{fs}$ fwhm broadening of the time resolution due to the different refractive indices for X-ray and optical light in water.

Though at a first approximation the shape of the difference spectra does not change too much with time delay, a closer inspection suggests a small spectral change that is especially noticeable around $7130 \mathrm{eV}$. Whereas at longer time delays the difference spectra cross zero at this energy, the difference signal for the first two or three are negative.

Previous studies ${ }^{11,12}$ had suggested that upon photoexcitation the system HS state is directly populated from the MLCT manifold. We have estimated the MLCT spectrum as follow. As described in detail in refs 11 and 12, upon the photoexcitation of $\left[\mathrm{Fe}(\mathrm{bpy})_{3}\right]^{2+}$ some of the electron density is shifted to the bpy ligands and thus the effective oxidation state of the central atom is changed from $\mathrm{Fe}$ (II) to $\mathrm{Fe}$ (III). As a consequence, the core hole shielding changes and more energy is required to excite a core electron with an X-ray photon, due to the fact that the molecular energy levels are shifted with respect to the deep-lying core levels. The XAS spectrum of a trivalent Fe would be expected to be shifted with respect to the one of the bivalent Fe. It has been suggested ${ }^{12}$ that to a first approximation, the MLCT spectrum could be represented as shifted copy of the ground state one. The amount of shift should be of the order of $2 \mathrm{eV}$. Using the ground state spectrum (LSs) and the HS-LS from ref 11, we calculated the MLCT spectrum for different edge shifts by translating the LSs to the predefined amount before interpolating on the original energy scale. The MLCT-LS spectra in the Figure 6 are then calculated as difference between the shifted spectra and the GSs.

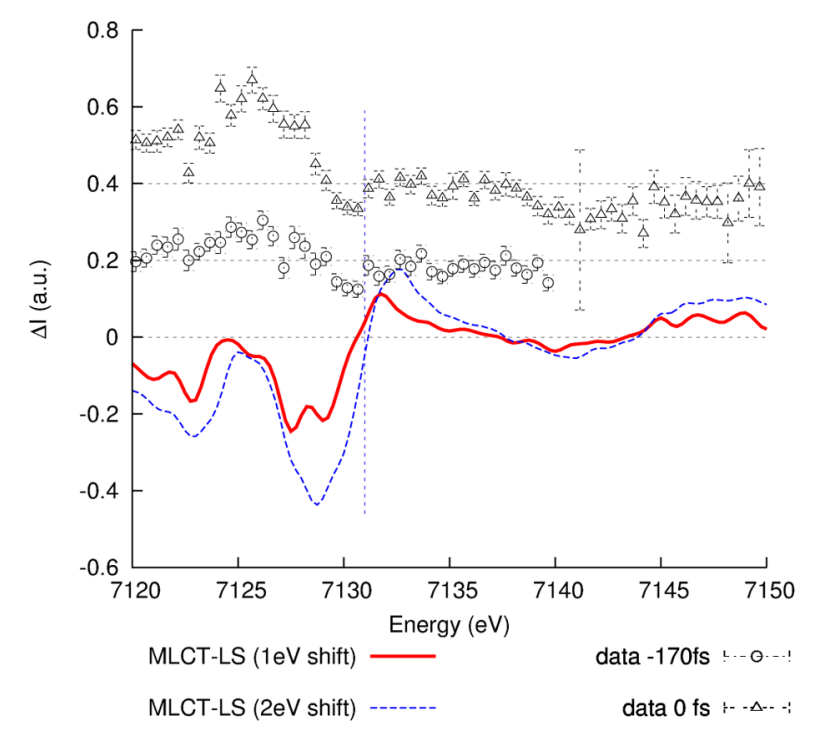

Figure 6. Difference spectra at the two shortest time delays (points) together with the calculated MLCT-LS spectrum as described in the text.

The MLCT contribution to the difference signal at $7125.5 \mathrm{eV}$ (time scan in Figure $5 b)$ is significantly smaller $(<10 \%)$ than the HS-LS difference spectrum. Therefore, with the present signal-to-noise ratio $(\mathrm{S} / \mathrm{N})$, the MLCT contribution cannot be resolved in the time scan of Figure $5 \mathrm{~b}$. The contribution of the MLCT and other intermediates is expected to be large compared to the HS-state contribution around $7131 \mathrm{eV}$ (dashed vertical line in Figure 5a), where the HS-LS difference spectrum has an isosbestic point (i.e., the difference spectrum crosses zero). Indeed, the spectral scans taken at the shortest time delay indicate a small blue shift of the zero-crossing and 
negative peak, which vanishes with increasing pump-probe time delay. The MLCT-LS model spectrum (calculated as described above) has a negative peak at $\sim 7128 \mathrm{eV}$ (Figure 6) that might be responsible for the apparent shift of the zero crossing as well as for the negative peak of our data. In view of the $\mathrm{S} / \mathrm{N}$ of the current data and the uncertainties in the model spectrum for the MLCT state, however, we cannot exclude that some other intermediate ligand-field state is responsible for the observed change of difference spectrum.

Our interpretation does not deviate from the previous femtosecond Fe K-edge XANES measurements on $\left[\mathrm{Fe}(\mathrm{bpy})_{3}\right]^{2+}$ by Bressler and co-workers ${ }^{12}$ and $\mathrm{Fe}$ L-edge XANES measurements of a related Fe spin-crossover complex $\mathrm{Fe}\left[\left(\operatorname{tren}(\mathrm{py})_{3}\right)\right]^{2+}$ by Huse and co-workers. ${ }^{14}$ Both studies showed instrument response time-limited dynamics for the formation of the HS excited state. However, in this study, higher $\mathrm{S} / \mathrm{N}$ and better time resolution allowed us to determine the formation time of the ${ }^{5} \mathrm{~T}_{2}$ state more precisely. Such improvement is made possible by ultrashort FEL pulses of high $\mathrm{X}$-ray flux, enabling to collect the data with high $\mathrm{S} / \mathrm{N}$ in much shorter time than with other pulsed $\mathrm{X}$-ray sources and in particular it allows the measurement of X-ray absorption spectra at different delays. Hence, a complete mapping of timeresolved spectra on the femtosecond time scale becomes possible. This is essential to disentangle the complex transient electronic and structural changes, where multiple intermediate excited states might play a role.

The most limiting factor in the presented data have been timing drifts (hundreds of fs per hour) that forced us to collect only few hundred shots per scan point to limit the duration of each scan to few minutes. A recently developed single shot timing diagnostics ${ }^{30}$ allows post-experiment jitter compensation and eliminate such timing drifts. This development will facilitate, together with thinner liquid jets, collecting the data with much higher signal-to-noise ratio and better time resolution in the near future that will provide new insights in ultrafast reactions.

\section{AUTHOR INFORMATION}

\section{Corresponding Author}

*E-mail: marco.cammarata@univ-rennes1.fr.

\section{Notes}

The authors declare no competing financial interest.

\section{ACKNOWLEDGMENTS}

This research was carried out at the Linac Coherent Light Source (LCLS) at the SLAC National Accelerator Laboratory. LCLS is an Office of Science User Facility operated for the U.S. Department of Energy Office of Science by Stanford University. K.H. and M.M.N. acknowledge funding from Danish National Research Foundation's Centre for Molecular Movies and DANSCATT. H.I. and K.H.K. were supported by the Research Center Program (CA1201) of IBS (Institute for Basic Science) and Creative Research Initiatives (Center for Time-Resolved Diffraction) of MEST/NRF in Korea. J.K. was supported by Inha University Research Grant (INHA-46438). K.J.G., R.W.H., and W.Z. acknowledge support from the AMOS program within the Chemical Sciences, Geosciences, and Biosciences Division of the Office of Basic Energy Sciences, Office of Science, U.S. Department of Energy. C.B., W.G., and A.G. were funded by the European XFEL. L.X.C. and A.B.S. acknowledge the support by the U.S. Department of Energy,
Office of Science, Basic Energy Sciences, under Contracts DEAC02-06CH11357.

\section{REFERENCES}

(1) Zewail, A. J. Phys. Chem. A 2000, 104, 5660-5694.

(2) Ihee, H.; Lorenc, M.; Kim, T. K.; Kong, Q. Y.; Cammarata, M.; Lee, J. H.; Bratos, S.; Wulff, M. Science 2005, 309, 1223-1227.

(3) Cammarata, M.; Levantino, M.; Schotte, F.; Anfinrud, P. A.; Ewald, F.; Choi, J.; Cupane, A.; Wulff, M.; Ihee, H. Nat. Methods 2008, 5, 881-886.

(4) Collet, E.; Lemée-Cailleau, M. H.; Cointe, M. B.-L.; Cailleau, H.; Wulff, M.; Luty, T.; Koshihara, S. Y.; Meyer, M.; Toupet, L.; Rabiller, P.; Techert, S. Science 2003, 300, 612-615.

(5) Schotte, F.; Lim, M.; Jackson, T.; Smirnov, A.; Soman, J.; Olson, J.; Phillip, G. J.; Wulff, M.; Anfinrud, P. Science 2003, 300, 1944-1947.

(6) Wöhri, A. B.; Katona, G.; Johansson, L. C.; Fritz, E.; Malmerberg, E.; Andersson, M.; Vincent, J.; Eklund, M.; Cammarata, M.; Wulff, M.; Davidsson, J.; Groenhof, G.; Neutze, R. Science 2010, 328, 630-633.

(7) Fritz, D. M.; et al. Science 2007, 315, 633.

(8) Johnson, S. L.; Vorobeva, E.; Beaud, P.; Milne, C. J.; Ingold, G. Phys. Rev. Lett. 2009, 103, 205501.

(9) Daranciang, D.; et al. Phys. Rev. Lett. 2012, 108, 087601.

(10) Chen, L.; Wang, Z.; Burdett, J.; Montano, P.; Norris, J. J. Chem. Phys. 1995, 99, 7958-7964.

(11) Gawelda, W.; Pham, V.-T.; Benfatto, M.; Zaushitsyn, Y.; Kaiser, M.; Grolimund, D.; Johnson, S. L.; Abela, R.; Hauser, A.; Bressler, C.; Chergui, M. Phys. Rev. Lett. 2007, 98, 057401.

(12) Bressler, C.; Milne, C.; Pham, V. T.; ElNahhas, A.; van der Veen, R. M.; Gawelda, W.; Johnson, S.; Beaud, P.; Grolimund, D.; Kaiser, M.; Borca, C. N.; Ingold, G.; Abela, R.; Chergui, M. Science 2009, 323, 489-492.

(13) Tomov, I.; Oulianov, D.; Chen, P.; Rentzepis, P. J. Phys. Chem. B 1999, 103, 7081-7091.

(14) Huse, N.; Cho, H.; Hong, K.; Jamula, L.; de Groot, F. M. F.; Kim, T. K.; McCusker, J. K.; Schoenlein, R. W. J. Chem. Phys. Lett. 2011, 2, 880-884.

(15) Schoenlein, R.; Chattopadhyay, S.; Chong, H.; Glover, T.; Heimann, P.; Shank, C.; Zholents, A.; Zolotorev, M. Science 2000, 287, 2237-2240.

(16) Emma, P.; et al. Nat. Photonics 2010, 4, 641-647.

(17) Creutz, C.; Chou, M.; Netzel, T.; Okumura, M.; Sutin, N. J. Am. Chem. Soc. 1980, 102, 1309-1319.

(18) Ordejòn, B.; de Graaf, C.; Sousa, C. J. Am. Chem. Soc. 2008, 130, 13961-8.

(19) Gutlich, P.; Goodwin, H. A., Eds. Spin Crossover in Transition Metal Compounds; Springer: Berlin/Heidelberg, Germany, 2004; pp 233-235.

(20) Bergkamp, M. A.; Chang, C. K.; Netzel, T. L. J. Phys. Chem. 1983, 87, 4441-4446.

(21) Gawelda, W.; Cannizzo, A.; Pham, V.-T.; van Mourik, F.; Bressler, C.; Chergui, M. J. Am. Chem. Soc. 2007, 129, 8199-8206.

(22) McCusker, J.; Walda, K.; Dunn, R.; Simon, J.; Magde, D.; Hendrickson, D. J. Am. Chem. Soc. 1993, 115, 298-307.

(23) Consani, C.; Prémont-Schwarz, M.; ElNahhas, A.; Bressler, C.; van Mourik, F.; Cannizzo, A.; Chergui, M. Angew. Chem., Int. Ed. Engl. 2009, 48, 7184-7187.

(24) de Graaf, C.; Sousa, C. Chem.-Eur. J. 2010, 16, 4550-4556.

(25) Brady, C.; McGarvey, J. J.; McCusker, J. K.; Toftlund, H.; Hendrickson, D. N. Spin Crossover in Transition Metal Compounds III; Topics in Current Chemistry; Springer: Berlin/Heidelberg, 2004; Vol. 235, pp 1-22.

(26) Feng, Y.; Feldkamp, J.; Fritz, D.; Cammarata, M.; Aymeric, R.; Caronna, C.; Lemke, H.; Zhu, D.; Lee, S.; Boutet, S.; Williams, G.; Tono, K.; Yabashi, M.; Hastings, J. Proce. SPIE 2011, 8140, 81400Q (6 pp).

(27) Margaritondo, G.; Ribic, P. R. J. Synchrotron Radiat. 2011, 18, $101-108$.

(28) Calculations that considered a $\mathrm{Si}(111)$ based monochromator and simulated LCLS spectra (with fixed central wavelength) suggested 
that the spiky nature of the spectrum should increase the relative fluctuations in going from white to monochromatic beam of about $20 \%{ }^{31}$ Because the measured increase is much bigger (from $15 \%$ to $125 \%$ ), central energy fluctuations contributed significantly to the observed intensity fluctuations. Recent upgrades of LCLS have reduced central energy fluctuations, thus lowering the relative fluctuations and increasing the average throughput of monochromatic beams. During more recent experiments a $\sigma_{I} / I$ of the order of $\sim 40-$ $50 \%$ could be achieved.

(29) In the meanwhile, the standard repetition rate of the LCLS machine has been increased to $120 \mathrm{~Hz}$.

(30) Harmand, M.; Coffee, R.; Bionta, M.; Chollet, M.; French, D.; Zhu, D.; Fritz, D.; Lemke, H.; Medvedev, N.; Ziaja, B.; Toleikis, S.; Cammarata, M. Nature Photon., accepted for publication.

(31) Lee, S.; Roseker, W.; Gutt, C.; Huang, Z.; Ding, Y.; Grübel, G.; Robert, A. Opt. Express 2012, 20, 9790-9800. 